\title{
Topologically controlled segmentation of 3D magnetic resonance images of the head by using morphological operators
}

\author{
Petr Dokládal $^{\mathrm{a}, 1}$, Isabelle Bloch ${ }^{\mathrm{a}, *, 2}$, Michel Couprie ${ }^{\mathrm{b}}$, Daniel Ruijters ${ }^{\mathrm{a}, 3}$, \\ Raquel Urtasun ${ }^{\mathrm{a}}$, Line Garnero ${ }^{\mathrm{c}}$ \\ ${ }^{a}$ Ecole Nationale Supérieure des Télécommunications, Département TSI-CNRS URA 820 and IFR 49, 46 rue Barrault, \\ 75013 Paris, France \\ ${ }^{\mathrm{b}}$ Ecole Supérieure d'Ingénieurs en Electronique et Electrotechnique, Laboratoire Algorithmique et Architecture des Systèmes \\ Informatiques BP. 99, Noisy-le-Grand 93162 Cedex, France \\ ${ }^{\mathrm{c}}$ LENA, CNRS UPR 640 and IFR 49, Hôpital La Salpétrière, 75651 Paris Cedex 13, France
}

Received 8 November 2001; received in revised form 6 March 2003; accepted 6 March 2003

\begin{abstract}
This paper proposes a new data-driven segmentation technique of 3D T1-weighted magnetic resonance scans of human head. This technique serves to the construction of individual head models. Several structures of the head are extracted. The morphology-oriented approach combined with an extensive use of topological constraints provides a robust and automatic method requiring minimum user intervention. This new approach is suitable to applications where the topology is one of the main constraints. The originality of the approach lies in the satisfaction of such constraints and in an effort towards robustness. (C) 2003 Pattern Recognition Society. Published by Elsevier Ltd. All rights reserved.
\end{abstract}

Keywords: Brain imaging; 3D segmentation; Mathematical morphology; Topological constraints

\section{Introduction}

Since anatomical brain imaging serves as a reference for clinical investigations as well as for functional studies, the segmentation of brain structures deserves a large attention and aims at many different applications, each imposing its

\footnotetext{
* Corresponding author. Tel.: +33-1-45-81-75-85; fax: $+33-1-45-81-37-94$.

E-mail addresses: dokladal@cmm.ensmp.fr (P. Dokládal), isabelle.bloch@enst.fr (I. Bloch), coupriem@esiee.fr

(M. Couprie), line.garnero@chups.jussieu.fr (L. Garnero).

${ }^{1}$ Current address: Ecole Nationale Supérieure de Mines de Paris, CMM, 35 rue Saint-Honoré, 77305 Fontainebleau Cedex, France.

${ }^{2}$ Thanks to RNRT Project COMOBIO for partial funding of this work. See http://www.tsi.enst.fr/comobio/

${ }^{3}$ Daniel Ruijters is now with Philips, Netherlands, and Raquel Urtasun is with EPFL, Switzerland.
}

own constraints on the segmentation. Here, the aim of the segmentation was to build $3 \mathrm{D}$ meshes of the main head tissues, where the respect of topology is of a prime importance. A large body of literature has been devoted to brain image segmentation (see e.g. the syntheses in Refs. [1-3]). In this paper, we will deal with T1-weighted magnetic resonance images (MRI). Segmentation methods can be distinguished depending on whether they make use of some models of the targeted structures or not. The models can be either implicit like deformable models or physics-based models, or explicit like atlas-deformation based techniques. Implicit models are often used when one specific structure of interest has to be detected. The atlas-based approaches can segment all the structures but have to deal with difficult problems due to the anatomical variability. Examples of the deformable models applied to the brain and brain structure segmentation can be found 
for instance in Refs. [4-8]. Sometimes, some statistical aspects are included in the model, which require some learning. Since such methods are usually applied to individual structures, it is difficult to guarantee the global topology when segmenting several tissues. Examples of atlas-based methods can be found in Refs. [9-17]. Methods that do not use any structure model employ mainly classification approaches. Many such methods have been developed to separate the main tissues. For instance, both fuzzy clustering (e.g. Refs. [18,19]) and neural networks (e.g. Refs. $[20,21]$ ) have been used, as well as probabilistic and statistical approaches (see for instance Ref. [1] for a review). Such methods rely on the grey level characteristics of the structures of interest, and can hardly incorporate other features, in particular topological ones.

Here, we are interested in methods that do not use any object model and that exploit not only the content of the image but also some constraints on the desired result, particularly topological constraints. Morphological methods are well adapted to this aim and provide natural tools for this. Most work using mathematical morphology in this domain concentrates on the segmentation of the brain and on the separation of the grey and the white matters (e.g. Refs. [2224]). Little attention was paid to the other structures until now. For instance, only few methods exist for the skull and the skin, and they usually rely on constraints different from those that we employ here (see e.g. Ref. [25]). For the separation of both hemispheres, most approaches rely on the optimization of a plane [26-29], or a template [30] which, however, does not necessarily respect well the shape of the separation. Here, based on a preliminary segmentation of the brain, we propose a morphological method to segment the brain stem and the cerebellum, the cerebrospinal fluid (CSF), the grey and white matters, the skull and the scalp. Furthermore, the two hemispheres are extracted separately and attention is paid to the grey/white matter interface, and to the grey matter/cerebrospinal fluid interface. The aim of this segmentation is to build from any standard MRI an individual 3D model of head structures which can serve for numerical solving of the electromagnetic wave propagation equations, as needed in electrophysiology, as well as when studying the influence of mobile phones on the head tissues. In contrary to other applications where a millimetric precision may be needed, for such applications the precision of the segmentation is not the key point. The most important aspect for the foreseen applications is to have a good and robust representation of the shapes and particularly preservation of the topology. The robustness is achieved in the proposed morphological approach by an intensive use of morphological reconstruction, conditional operations and by reducing the number of parameters. The topology is controlled by using homotopic transformations. An important aspect of the proposed method is the completely automatic selection of markers that limits the necessary user interaction and which also increases robustness. The method has been applied to thirteen 3D MR images from different acquisition devices (T1-weighted MRI), and has always provided good results.

The paper is organized as follows. In Section 2 we recall some basic notions related to homotopic morphological operations. We propose a way to select automatically a given number of markers. We outline briefly the principle of the homotopic deformations that combine topological and other criteria (on distance or on grey levels for instance). In Section 3 , we present successively methods for segmenting the structures of interest, which are applications on real cases of theoretical results of digital topology. The experiment results are given in Section 4 as well as some comments on the parameter estimation and robustness.

\section{Morphological operators under robustness and topological constraints}

We use the following notations. Let $f: \mathbb{Z}^{3} \rightarrow \mathbb{N}$ denote a digital image. Let $\delta_{\Gamma}(f)$ denote a dilation of $f$ by a structuring element $\Gamma, \Gamma \subset \mathbb{Z}^{3}: \delta_{\Gamma}(f)(x)=\bigvee_{x_{i} \in \Gamma(x)} f\left(x_{i}\right)$, where $\Gamma(x)$ denotes the translation of $\Gamma$ at $x$ [31] whereas $\varepsilon_{\Gamma}(f)$ denotes the erosion of $f$ by $\Gamma$ defined as $\varepsilon_{\Gamma}(f)=$ $\bigwedge_{x_{i} \in \Gamma(x)} f\left(x_{i}\right)$. The symbol $\gamma_{\Gamma}(f)=\delta_{\Gamma}\left[\varepsilon_{\Gamma}(f)\right]$ denotes the opening and $\varphi_{\Gamma}(f)=\varepsilon_{\Gamma}\left[\delta_{\Gamma}(f)\right]$ the closing (these definitions are for symmetrical structuring elements, which will always be the case in the following; in the general case, one of both operations has to be performed with the symmetrical of the structuring element). The set-wise version of dilation is obtained from the dilation on function by replacing sup (supremum) by $\cup$ (union) and inf (infimum) by $\cap$ (intersection). For any subset $X$ of $\mathbb{Z}^{3}, \bar{X}$ denotes the complement of $X$. The function dist : $\mathbb{Z}^{3} \times \mathbb{Z}^{3} \rightarrow \mathbb{N}$ denotes an approximation of the Euclidean distance in $\mathbb{Z}^{3}$ [32]. We use the same notation for the distance from a point to a set. The function dist ${ }^{X}$ denotes the geodesic distance, calculated within the set $X$. For any point $x \in \mathbb{Z}^{3}, N(x)$ denotes some neighborhood of $x$, such that $N(x) \subset \mathbb{Z}^{3}$ (it can be typically the elementary neighborhood related to the 6-, 18-, or 26-connectivity). The operator $\operatorname{bd}(X)$ denotes the boundary of $X: \operatorname{bd}(X)=\{x \mid x \in X$ and $N(x) \cap \bar{X} \neq \emptyset\}$. Thereby, the boundary of any set is a subset defined as the union of the points $x$, included in the set, such that their neighborhood intersects both the set and its complement. On the other hand, the interface is an anatomical concept specifying the common face of two adjacent organs. It does not have a rigorous geometric meaning.

\subsection{Morphological reconstruction}

Morphological reconstruction is one classical way in mathematical morphology to increase the robustness of operations. The principle consists in conditioning a chosen transformation to some reference set or image, and controlling thereby its spatial extension [31,33]. Geodesic transformations are defined by using balls of the geodesic 
distance as structuring elements. In the digital case, the geodesic dilation of a set $Y$ conditionally to a set $X$ (with $Y \subseteq X)$ by a ball of radius 1 is simply computed as [33]: $\delta^{X}(Y)=\delta(Y) \cap X$, where $\delta^{X}$ uses the geodesic ball of radius 1 as a structuring element. The morphological reconstruction $\rho^{X}(Y)$ of $Y$ in $X$ is then defined by iterating this conditional dilation until convergence: $\rho^{X}(Y)=\left(\delta^{X}\right)^{\infty}(Y)$, with $\left(\delta^{X}\right)^{n}(Y)=\delta^{X}\left[\left(\delta^{X}\right)^{n-1}(Y)\right]$ (note that convergence is achieved in a finite number of steps for $X$ finite). These definitions extend to functions and the morphological reconstruction of $f^{\prime}$ under $f$ is defined as $\rho^{f}\left(f^{\prime}\right)=\left(\delta^{f}\right)^{\infty}\left(f^{\prime}\right)$, with $\delta^{f}\left(f^{\prime}\right)=\delta\left(f^{\prime}\right) \wedge f$. The other geodesic morphological operations are defined in a similar way.

\subsection{Bottleneck constriction}

We call a bottleneck constriction the splitting of one connected component into several objects, the separation occurring at the narrowest junctions. A narrow junction in some object $X$ is identified as a topographic saddle of the distance function $g(x)=\operatorname{dist}(x, \bar{X})$. One would expect that the splitting of $X$ into several objects can be done in $g$ by searching for regional maxima followed by a watershed reconstruction. In real cases (noisy objects, fragmented borders, etc.), however, we may obtain many more maxima than the number of significant objects. Instead, we have to consider only the maxima corresponding to (or marking) the significant objects according to some suitable criterion of selection and rejection of markers. The objects are then obtained by reconstruction of the chosen markers in the distance function. In a general case, where no topological assumptions are made, one may use the watershed transform (see e.g. Ref. [34]) for this reconstruction. In situations where the topology of the result needs to be controlled, we may use a reconstruction based on a homotopic deformation of the markers. If the markers have the correct topology, then the topology of the reconstructed objects will remain unchanged. In the following section we propose a new completely automatic marker selection scheme. This scheme permits to control the number of resulting objects and select those that maximize some chosen criterion.

\subsection{Component tree and automatic selection of markers}

A direct application of watershed algorithms often provides a poor segmentation result. Indeed, every function minimum gives birth to one segment in the result. The input image often contains a multitude of minima (several of them being not significant) which bring over an oversegmentation of the input image. Several methods can be used to avoid this, consisting mainly in filtering either the initial image or the image on which the watersheds are applied (typically a gradient image), or merging a posteriori the obtained regions. If some a priori knowledge of the desired segments is available then one may employ a powerful method using markers. The markers will limit the number of the func-

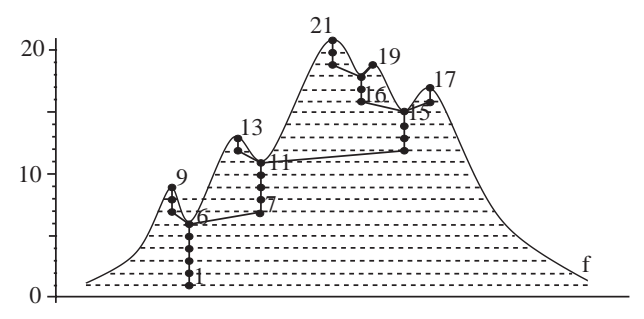

Fig. 1. 1-D example of function $f$ and the component tree (the values represent the thresholding level giving birth to the component; not all values are given).

tion minima before the watersheds are applied. Then the watershed-based segmentation techniques (see for instance Ref. [34]) are usually based on the following scheme:

(1) selection of markers of the objects to extract,

(2) reconstruction of boundaries between the marked objects by using the watersheds.

The selection of markers can be done in various ways, depending on the type of available knowledge. For our application, we exactly know the number of objects to find. Moreover, we want to define them as the most pertinent areas of the grey level function, according to some criterion. If this approach were implemented in a naive way, it would require to try all the possible values of this criterion, until the right number of objects is found. We propose here a new way to extract a known number of markers maximizing this criterion with a reasonable time complexity. We use a component-tree representation of a function towards this aim. The use of a tree in order to represent the "meaningful" information contained in a function is not new. In particular, Hanusse and Guillataud [35] claim that such a tree can play a central role in image segmentation, and suggest a way to compute it, based on an immersion simulation. Several authors, such as Vachier [36], Breen and Jones [37], Salembier et al. [38] have used this structure for efficient implementation of some morphological operators (e.g. attribute openings, granulometries, extinction functions). The algorithms used to compute the component tree can be found in Refs. [37-39]. The last reference also contains a discussion about the time complexity of different algorithms. Let $f$ denote a function $f: \mathbb{Z}^{3} \rightarrow \mathbb{N}$ and let $k \in \mathbb{N}$. We consider a binarization of $f$ obtained by thresholding $f$ at level $k: f^{k}=\{x \mid f(x) \geqslant k\} . \mathscr{C}^{k}(f)$ denotes the set of all connected components of $f^{k}$ and $\mathscr{C}(f)$ is the set of components obtained with all possible $k$. These components for all possible values of $k$ are organized in a directed component tree (see Fig. 1) defined as:

(1) the vertices of the tree are the elements of $\mathscr{C}(f)$,

(2) the arcs represent the inclusion relations between the corresponding components. The relations that can be obtained by transitivity are omitted. 
Attributes: To each component in $\mathscr{C}(f)$, we can associate numbers which measure some of the component characteristics. In the following, $\# X$ denotes the cardinal of the set $X$. Let $k \in \mathbb{N}$, let $c \in \mathscr{C}_{k}(f)$, we define:

- $h(c)=\max \{f(x)-k+1, x \in c\}$, the height of the component $c$;

- $a(c)=\# c$, the area of the component $c$;

- $v(c)=\sum_{x \in c}(f(x)-k+1)$, the volume of the component $c$ (i.e. the volume delimited by the function and the horizontal plane at height $k$ ).

The mappings $h, a, v$ are called attributes. These three attributes are increasing (with respect to the inclusion relation): we say that an attribute $\alpha$ is increasing if $\forall c, c^{\prime} \in \mathscr{C}(f)$, $c \subseteq c^{\prime} \Rightarrow \alpha(c) \leqslant \alpha\left(c^{\prime}\right)$. Suppose that, for a given function $f$, we need to find the $N$ most significant lobes with respect to either the height, area or volume criterion. These lobes will serve as markers for subsequent bottleneck constriction algorithm. By using the tree, this task reduces to the search of the $N$ vertices that have the largest attribute values and are not bound (even transitively) by the inclusion relation. The algorithm reads as follows:

Compute the component tree $T$ for the function $f$;

Compute the attribute values for the vertices of $T$

(height, area or volume);

Sort the vertices of $T$ by increasing order of

attribute value;

Count the number $L$ of leaves in $T$;

While $L>N$ do

Choose a (leaf) vertex $c$ in $T$ with smallest attribute value;

Remove $c$ from $T$ (keeping unchanged the attribute values of other vertices);

If this removal does not create a new leaf in $T$, then do

EndWhile

$$
L=L-1 \text {. }
$$

The remaining leafs (more precisely, the pixels which are associated to these leafs) constitute the desired markers. An illustrative example is given in Fig. 2. Suppose that the desired segmentation contains two objects as given in Fig. $2 d)$. For the segmentation we use the height attribute. The above algorithm is initialized with $N=2$ desired resulting components. Initially, the tree contains $L=11$ leaves. The algorithm iteratively suppresses the leaves with the smallest weights: $1,2,3,5,6,7,9,10,12,13,14$ (all of them having the weight equal to 1 ), until there are only two leaves left in the remaining tree: 8 and 11 (with weights 21 and 11 , respectively). Using various attributes gives birth to different segmentations even if the same number of resulting objects is specified. Consider a digital function $f$ the values of which are given in Fig. 3. For $k=1$ we obtain three connected components for the set $\{x \mid f(x) \geqslant k\}$. The height is equivalent for all of the three components, the second and

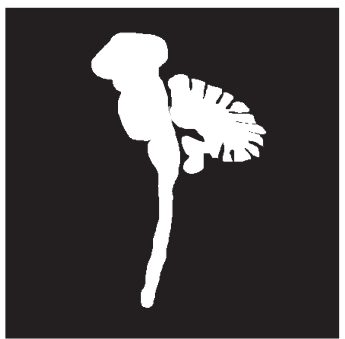

(a)

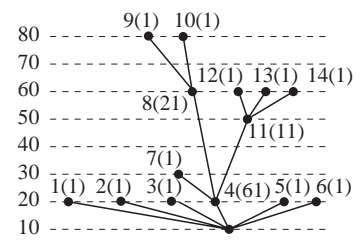

(c)

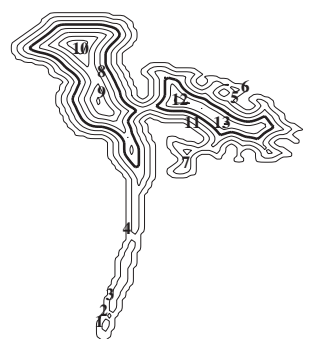

(b)

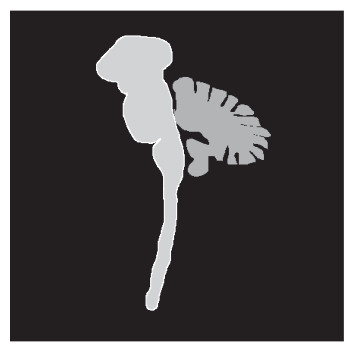

(d)
Fig. 2. Segmentation by bottleneck constriction: (a) 2D input object, (b) contours of distance to the complement with labeled connected components, (c) corresponding component tree with labeled vertices and the height-attribute values in parentheses, (d) segmentation in two most pertinent objects.

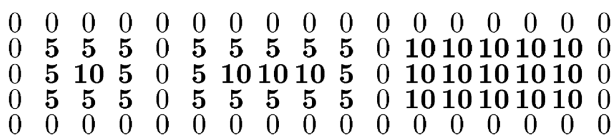

component:

height:

area:

1
10
9
50

2

15

90

3
10
15
150

Fig. 3. Example of a function $f$. For $k=1, f^{k}$ contains three connected components (given in bold). The height, area and volume attribute values are given below.

the third components have the same area. The volumes differ for all the three components. The level $k$ and the area $a$ of the nodes have a special geometrical meaning when used with a distance function. Consider some object $X$ and some function $g$ representing the distance to the complement $\bar{X}$. Consider a node $c_{i}$ of the component tree of $g$, corresponding to some component of $g^{k}$, i.e. $\left(c_{i} \in \mathscr{C}^{k}(g), k \in \mathbb{N}\right)$. Hence, a ball of radius $k$ centered somewhere in $c_{i}$ is necessarily included in $X$. The level $k$ corresponds to the radius of the maximal included ball and the area $a(c)$ to the number of all such balls. Note that this property assumes that the digital distance is chosen in accordance with the connectivity used for defining the connected components. The automatic selection of markers used in this approach relies on the (arbitrary but known) number of resulting objects. The 
function $g$ is some distance function. The selected markers are those maximizing either the criterion of maximal included ball (for the height attribute) or the number of maximal included balls, if the radius is equal for several components (for the area attribute). We have observed a significant increase in the segmentation robustness for the area and volume attributes compared to the height, as shown in the experiment section.

\subsection{Homotopic transformations under constraints}

Homotopic transformations can be performed in digital spaces thanks to the use of simple points (e.g. $[40,41])$, i.e. points that can be successively deleted from a set $X$ (or added to $X$ ) without modifying its topology. Note, however, that simultaneous deletion of several simple points may change the topology. Therefore, any transformation which acts exclusively by sequential deletion or addition of simple points is homotopic. In order to guarantee a topologically consistent result, we extensively use homotopic deformations throughout the segmentation process. Although thinning and thickening are very popular in image processing and digital topology, here we use more recent tools, where these operations are constrained not only by topological criteria but also other ones such as grey levels, distances, etc. They are called $\lambda$-thinning and $\lambda$-thickening and were first introduced in Refs. [42,43]. These operators perform a homotopic thinning (respectively thickening) of some binary object according to some criterion $\lambda$. The $\lambda$-thickening is defined by the following algorithm:

$$
\begin{aligned}
& \text { Reconstruction by } \lambda \text {-thickening } \\
& \mathrm{X}:=\text { marker } \\
& \text { repeat until stability } \\
& \quad \text { select a simple point } x \text { satisfying } \lambda \\
& \quad X:=X \cup\{x\}
\end{aligned}
$$

The criterion $\lambda$ can be of any nature, not necessarily a topological one. $\lambda$-thickening allows to perform transformations combining topological constraints with any other constraint. Moreover, it is known that the result of thinning or thickening depends on the order in which the points are tested. If $\lambda$ introduces an order on the set of points then the selection of a simple point may be determined by this order. The priority will be given by the degree of satisfaction of $\lambda$. For instance, $\lambda$ can be a criterion on grey level, as $f(x)<C$, and points are ordered according to their grey level. Let $X$ denote some initial binary object and $f$ a grey-valued image; the $\lambda$-thickening iteratively adds to $X$ all simple points such that $f(x)<C$. The darkest simple points are added first. The deformation process stops as soon as there are no more simple points such that $f(x)<C$. The $\lambda$-thinning operator is derived from $\lambda$-thickening by duality with respect to complementation, and iteratively deletes points from $X$ according to the criterion $\lambda$. Throughout the segmentation process, the object markers found by the component tree are often reconstructed by using one of the $\lambda$-reconstructions. The construction of the component tree is actually similar to the extraction of a watershed by flooding where all the local minima represent the markers. During the flooding we construct the tree while associating the component labels and the attribute values. The advantage is that once the tree is constructed, the search of different components by using various types of criteria or even different number of components becomes very fast. During the reconstruction of the objects from the markers either morphological or homotopic reconstruction can be used. We prefer $\lambda$-reconstructions over morphological reconstructions for their ability to preserve the topology provided that the markers already have the topology of the final object.

\subsection{Cavity and hole}

In binary images we call background the complement of the object. A cavity denotes a connected component of the background which is not adjacent to the border of the image. For example, the interior of a hollow sphere is a cavity. In 3D binary images, a hole denotes intuitively a tunnel through an object. For example, a solid torus has a hole. Contrarily to a cavity, the concept of hole does not exist in 2D. Note that an object containing holes and/or cavities cannot be reduced to a single point by a series of homotopic operations. While the filling of cavities is rather a trivial operation both in 2D and $3 \mathrm{D}$, which can be performed by using labeled background components, the closing of holes has been proposed only recently in Ref. [44]. The hole closing algorithm described below is a simplified version of this method, suited to the case of connected objects. The hole closing algorithm is initialized by a bounding box $B$ of the object containing holes $X, B \supset X$. The bounding box $B$ is submitted to $\lambda$-thinning controlled by the distance function $d(x)=\operatorname{dist}(x, X)$. The bounding box is a simply connected object with no holes nor cavities and $\lambda$-thinning cannot create holes. The thinning $Y=\lambda$-thinning $(B)$, where $\lambda$ is $d>0$, stops as soon as there are no more simple points to delete (by construction $Y$ is without holes). At the same time, no point $x \in X$ can be deleted since $d(x)=0$ for all $x \in X$. Although, during the thinning, preference is given to points more distant from the object $X$, counter-examples have been found where the holes are not closed with the minimal surface. In the sequel, Fill $(X)$ denotes the operator filling up the holes and cavities of $X$.

\section{Segmentation method}

In this section we describe the segmentation process. The structures are extracted one by one in the order they are listed below. In the text, we use numerical indices for the intermediate objects and abbreviations for complete organs. 

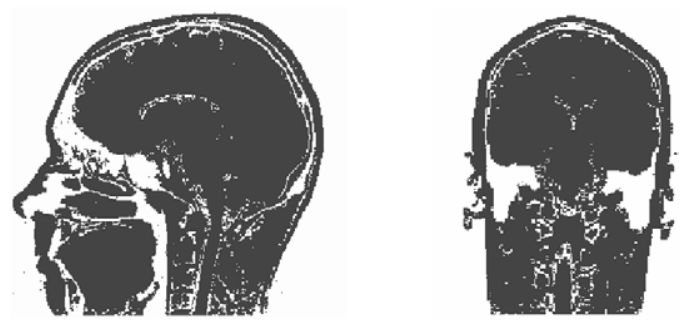

Fig. 4. Result of the initial thresholding (object $X_{1}$ ). The CSF, bone and fat have been eliminated. The $3 \mathrm{D}$ process is illustrated here on two orthogonal slices (sagittal and coronal).
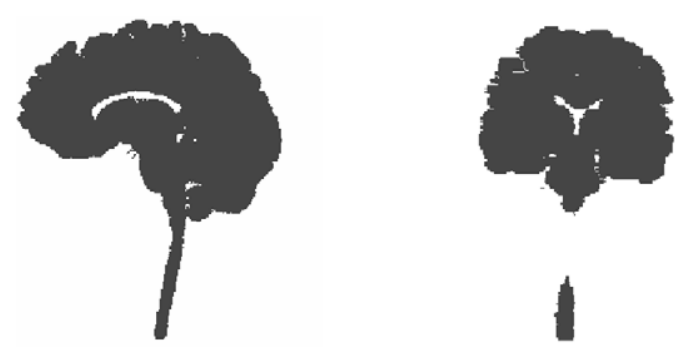

Fig. 5. The encephalon reconstructed from the marker (object $X_{9}$ ).

\subsection{Encephalon}

The extraction of the encephalon mask has been developed in Ref. [45]. The result of this stage $-X_{E N C E P H}-$ is a smooth envelop of the brain including the cerebrospinal fluid. The cerebellum, brain stem, hemispheres and the cerebrospinal fluid are then extracted from the encephalon mask. Let $f$ be the initial grey-scale image. The initial thresholding $X_{1}=\left\{x \mid t_{1}<f(x)<t_{2}\right\}$, where $t_{1}=\mu_{C S F}-\left(\sigma_{C S F} / 2\right)$ ( $\mu_{C S F}$ and $\sigma_{C S F}$ being the mean and standard deviation of the grey levels in the CSF) and $t_{2}=\mu_{F A T}-\sigma_{F A T}\left(\mu_{F A T}\right.$ and $\sigma_{F A T}$ being the mean and standard deviation of the grey levels of fat), eliminates the dark elements (the lower limit) as the cerebrospinal fluid, bone and air and the vascularization and fat (the upper limit). The choice of the lower limit is essential for good disconnection of the encephalon from other structures of the head. However, it is not difficult to find the good value since the tissues are usually well contrasted. The actual disconnection is performed by the erosion: $X_{2}=\varepsilon X_{1}$ where the structuring element is a ball with a radius $R=4$ to $5 \mathrm{~mm}$. Fig. 4 gives the object $X_{1}$. Let $X_{3}$ be the largest component of $X_{2}$. The component $X_{3}$ then marks the encephalon. The eroded encephalon is reconstructed by dilation $X_{4}=\rho^{X_{2}}\left(X_{3}\right)$. The structuring elements used for the reconstruction and the previous erosion are the same, see Fig. 5. A $3 \mathrm{D}$ binary closing $X_{5}=\varphi_{\Gamma}\left(X_{4}\right)$ will close the cortical furrows and ventricles and smooth the encephalon surface. The structuring element $\Gamma$ is a ball with a radius $R=5 \mathrm{~mm}$. Finally, the hole and cavity filling is applied to fill up the cavities $X_{E N C E P H}=\operatorname{Fill}\left(X_{5}\right)$, see Fig. 6 .
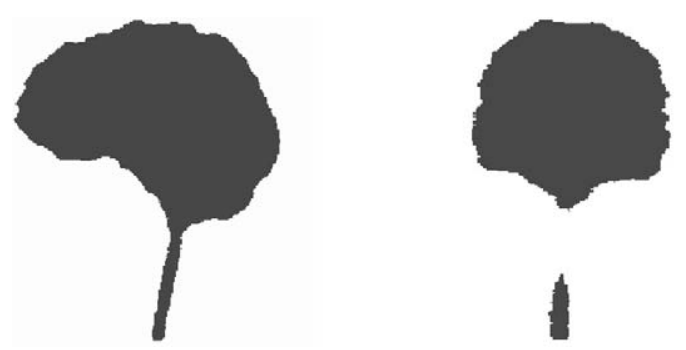

Fig. 6. The encephalon mask $X_{E N C E P H}$.

\subsection{Brain stem, cerebellum and cerebrum}

The segmentation of the cerebellum and the brain stem is generally not addressed in the literature. We base it on the bottleneck constriction (see Section 2). These objects are first automatically separated from the encephalon and then from each other at the narrowest junction situated in the mesencephalon and in the cerebral peduncles. In the following, the function $f$ denotes the initial MRI. First, we obtain an intermediate object: $X_{6}^{\prime}=\left\{x \mid x \in X_{E N C E P H}\right.$ and $\left.f(x)>s_{1}\right\}$, where $s_{1}=\mu_{\text {CORTEX }}$ is the mean value of the grey matter (see Section 4 for the automatic estimation of the parameters). From now on, we only consider the lower part of $X_{6}^{\prime}$ limited on the top by the higher extremity of the tegmentum. This can be easily found manually. Let $X_{6}$ denote the resulting object. The separation proceeds in two steps. First, we identify the lateral hemisphere lobes which will be deleted, then we separate the brain stem from the cerebellum. By using the component tree (Section 2) we find three markers $M^{\prime}=$ $\left\{M_{1}, M_{2}, M_{3}\right\}$ corresponding to the three most significant lobes of $g_{1}(x)=\operatorname{dist}\left(x, \overline{X_{6}}\right)$. Since the searched objects are simply connected with no cavities, the markers are submitted to a cavity and hole filling algorithm [44] $M=\operatorname{Fill}\left(M^{\prime}\right)$. The objects are then obtained by homotopic reconstruction of the three markers in $g_{1}: X_{7}=\lambda$-thickening $(M)$, where $\lambda$ is $g_{1}(x)>0$. The largest connected component of $X_{7}$ gives the union of the cerebellum and the brainstem: $X_{B S+C B}$. The second step separates the brainstem and the cerebellum. The separation is based on the bottleneck constriction of the morphological closing of $X_{B S+C B}$. This closing regularizes the laminar structure of the cerebellum which would have otherwise perturbed the distance function. The resulting smooth-surfaced object is denoted by $\varphi_{\Gamma}\left(X_{B S+C B}\right)$. The structuring element $\Gamma$ is a ball of radius $R=1 \mathrm{~mm}$. We search three markers $M^{\prime}=\left\{M_{1}, M_{2}, M_{3}\right\}$ marking the most significant lobes of $g_{2}$, calculated as $g_{2}=\operatorname{dist}\left(x, \overline{\varphi_{\Gamma}\left(X_{B S+C B}\right)}\right)$. After a rectification of the topology $M=\operatorname{Fill}\left(M^{\prime}\right)$, by homotopic reconstruction of $M$ we obtain $X_{8}=\lambda$-thickening $(M)$, where $\lambda$ is $g_{2}(x)>0$. The brainstem is the smallest connected component of $X_{8} \cap X_{B S+C B}$. The cerebellum $X_{C B}$ is obtained as the union of the two largest connected components of $X_{8} \cap X_{B S+C B}$. The intersection permits to recover the laminar aspect of the cerebellum. Finally, we obtain the 


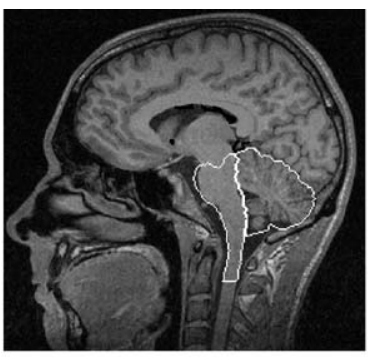

(a)

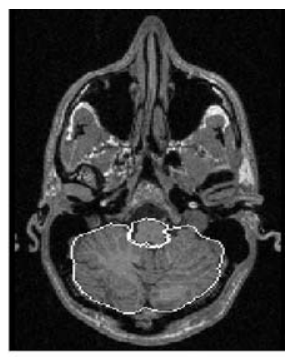

(b)
Fig. 7. Contours (in white) of the cerebellum $X_{C B}$ and the brain stem $X_{B S}$, sagittal and axial views.

cerebrum by $X_{\text {CEREBRUM }}=X_{E N C E P H} \backslash X_{B S} \backslash X_{C B}$. The proposed method uses the a priory knowledge we have about the number of objects of interest, and the automatic selection of the corresponding number of markers, chosen as the most significant ones (Fig. 7).

\subsection{Cerebrospinal fluid}

This method extracts the object $X_{C S F}$ representing the CSF both in the ventricles and in the sulci. We impose to the resulting $X_{C S F}$ the topology of a hollow sphere: it corresponds to the reality but not necessarily to the input image because of its limited resolution. This is a specificity of the proposed approach, to include constraints related to reality even if the data do not exactly satisfy them. We start with an initial thresholding: $X_{9}=\left\{x \mid x \in X_{E N C E P H}\right.$ and $\left.f(x)<s_{2}\right\}$ where $s_{2}=\mu_{C S F}+2 \sigma_{C S F}$, and $\mu_{C S F}$ and $\sigma_{C S F}$ are the mean and the standard deviation of CSF. Then we filter out the noisy points by morphological opening: $X_{10}=X_{9} \backslash \gamma_{\Gamma}\left(X_{9}\right)$ where $\Gamma$ is a ball of radius $R=1 \mathrm{~mm}$. We define a new grey-scale image $g$ as

$g(x)=\left\{\begin{array}{ll}f(x) & \text { if } x \in X_{10}, \\ s_{2} & \text { if } x \in X_{9} \\ 0 & \text { otherwise. }\end{array}\right.$ and $x \notin X_{10}$,

The points from $X_{10}$ are reported from $f$ with the same grey-level value, these points are likely to belong to the cerebrospinal fluid. Isolated points or points belonging to small structures, filtered out by the opening above, get a new value $s_{2}$. (These points would also belong to the CSF but they are likely to be just noise since they are isolated.) Indeed, the grey-level $s_{2}$ is the lowest value authorized for the reconstruction of the CSF complement below. Hence, these points will not be part of the CSF. We extract $X_{C S F}$ by reconstruction of its complement in $X_{E N C E P H}$ denoted as $\overline{X_{C S F}}$. Let $X_{11}=\lambda$-thickening $(M)$, where $\lambda: g(x) \geqslant s_{2}$, and the marker $M$ is an arbitrary point of the encephalon $X_{E N C E P H}$ such that $f(x)>s_{2}$ (i.e. in $\overline{X_{C S F}}$ ). The CSF is then obtained by subtraction: $X_{C S F}=\delta_{\Gamma}\left(X_{E N C E P H}\right) \backslash X_{11}$, where $\Gamma$ is a ball of radius $R=1 \mathrm{~mm}$ (see Fig. 8). The dilation guarantees that
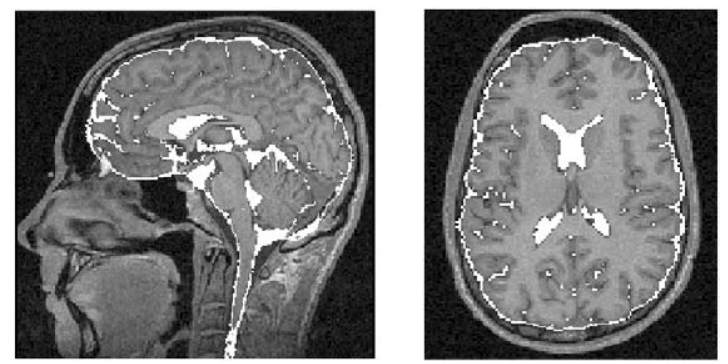

Fig. 8. The cerebrospinal fluid $X_{C S F}$ (in white), sagittal and axial views.

$X_{C S F}$ contains all CSF. Note that the dilation $\delta_{\Gamma}$ by a ball of $R=1 \mathrm{~mm}$ cannot (the absorption law, see [46]) modify the topology of $X_{E N C E P H}$ since this object has previously been smoothed by a closing with a ball of $R=10 \mathrm{~mm}$ [47]. The topology of $X_{C S F}$ is a hollow sphere. From now on, we consider $X_{E N C E P H}$ as $X_{E N C E P H} \backslash X_{C S F}$.

\subsection{Grey and white matters}

The segmentation of the grey and white matters is initialized from the classical $k$-means classification algorithm used to identify the intensity clusters of the two tissues. We obtain the cluster-separation threshold $c_{1}=k$-means $\left(f \cap X_{C E R E B R U M}\right)$ with $k=2$ for two clusters. The interface between the white and the grey matters is obtained by $\lambda$-thickening. First, we identify the largest component of the white matter $X_{8}$ by taking $X_{7}=\left\{x \mid x \in X_{\text {CEREBRUM }}\right.$ and $\left.f(x)>c_{1}\right\}$ and $X_{8}$ the largest connected component of $X_{7}$ which will, after the filling of cavities $M=\operatorname{Fill}\left(X_{8}\right)$, serve as marker for $\lambda$-thickening. The $\lambda$-thickening is controlled by the geodesic distance $g$ to the marker, conditionally to the complement of the grey matter by $g(x)=\operatorname{dist}^{X_{7}}(x, M)$. The white matter $X_{W M}$ is then reconstructed homotopically from the marker $M$ as $X_{W M}=\lambda$-thickening $(M)$, where $\lambda$ is $g<\infty$. Note that the $\lambda$-reconstruction is limited only to points classified as white matter since $g(x)=\infty$, for all $x \notin$ $X_{7}$. During the reconstruction the priority is given to simple points which are at the smallest geodesic distance from the initialization. The interface between the grey matter and the CSF is obtained in a similar way, by $\lambda$-thickening in the complement of CSF, with the same priority depending on geodesic distances. These ideas are detailed below. The human anatomy may vary substantially between different individuals, and this is particularly true for the cortex. On the other hand, the topology always remains unchanged despite the differences in the shape and the structure. Again, the foreseen applications led us to privilege the topology over the millimetric precision. The cerebral cavities (e.g. the ventricles) and convolutions are particularly sensitive to the partial volume effect, especially in regions where the space between two adjacent gyri gets very small. Because of the partial volume effect the gyri sometimes seem to grow 


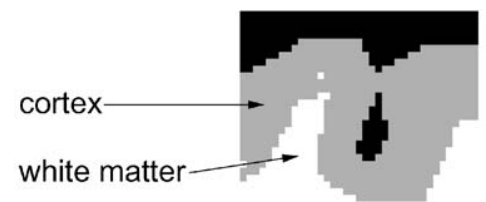

(a)

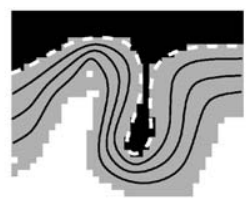

(b)

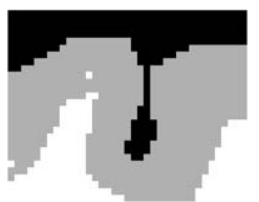

(c)

Fig. 9. Extraction of the white matter and the cortex: (a) an originally open concavity appears closed due to partial volumes, (b) the dashed line indicates the real cortex surface; the solid lines indicate the distance isovalues used to control the $\lambda$-reconstruction of the cortex from $X_{W M}$, (c) correctly segmented concavity remains open.

together, whereas in reality this is never the case. Consequently, the topology of the surface, found in such a MRI scan, might be compromised. We will try to solve this in our approach. It may seem that the easiest method to obtain the cortical surface would be to simply extract the boundary of the volume representing the cerebrum $\operatorname{bd}\left(X_{C E R E B R U M}\right)$. However, as this method does not control the topology, it does not necessarily deliver the required topology. The principal cause is the partial volume effect. We propose a different approach. The cortex is mainly a layer of almost constant thickness covering the white matter. We start with the simply connected component (without any hole or cavity) representing the white matter $X_{W M}$ and perform a $\lambda$-thickening conditionally to $X_{\text {CEREBRUM }}$. The grey matter is obtained as $X_{\text {CORTEX }}=\lambda$-thickening $\left(X_{W M}\right) \backslash X_{W M}$, where $\lambda$ is $g<\infty$, where $g(x)=\operatorname{dist}^{X_{C E R E B R U M}}\left(x, X_{W M}\right)$. Note that the cortex reconstruction is limited to points $x \in X_{C E R E B R U M} \backslash X_{W M}$. This allows us to add progressively the points that are inside the

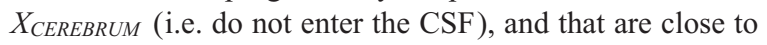
the white matter while the topology ( $X_{\text {CORTEX }}$ is one connected component with one cavity due to the subtraction of $X_{W M}$ ) remains preserved. This method guarantees that the sulcus concavities, which may appear closed due to the partial volume effect, are kept open. This topology corresponds best to the real cortical surface (see Fig. 9). Fig. 10 shows a few axial slices; the cortical surface is represented by white closed contours. Note that the disconnections are only due to the $2 \mathrm{D}$ representation. The $3 \mathrm{D}$ surface is actually closed. Fig. 11 shows two 3D renderings of the cortical surface. The quality of the cortical surface depends heavily on the quality of the segmentation of the parts that are taken as input (brain-mask, cerebellum, brainstem, CSF and white matter). If, for instance, the cerebrospinal fluid is not segmented correctly, the cortical surface that is found by our approach will not have a correct form either. Here, thanks to the robustness of our approach, this problem does not occur. The extraction of the grey/white matter interface is to some extent equivalent to the following three steps: (1) finding the markers by identifying the most prominent maxima in the distance to the complement, followed by (2) applying the watershed and (3) some topology rectification step. However, the white matter and the cortex have complex geometric forms due to numerous giry and furrows

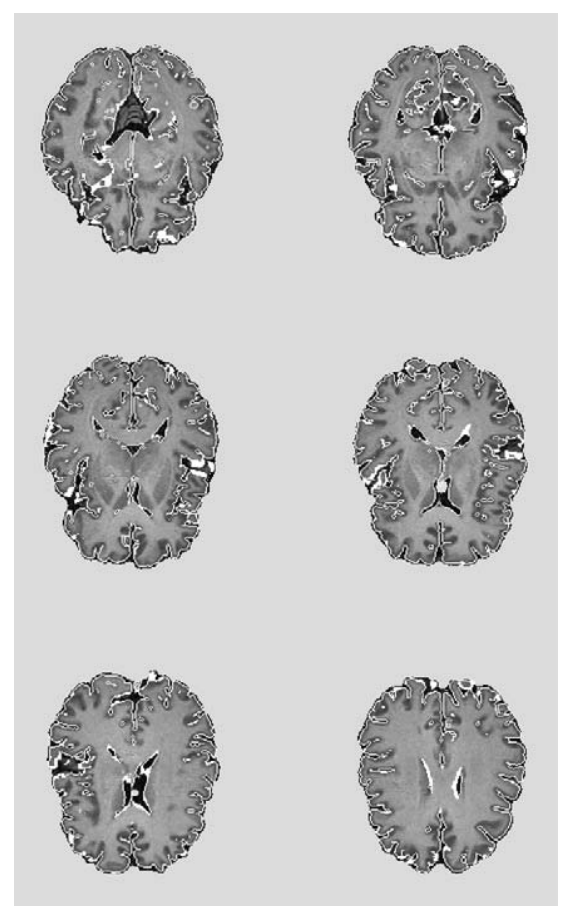

Fig. 10. The cortical surface in white, superimposed on a few slices.
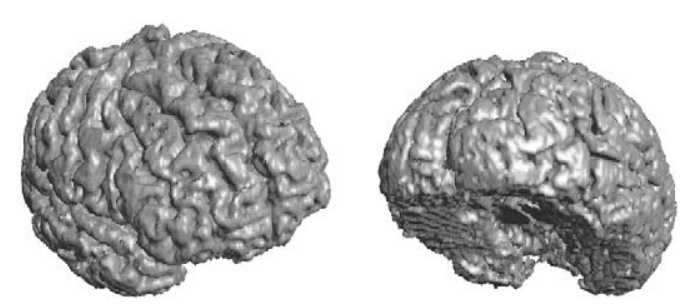

Fig. 11. 3D representation of the cortical surface, upper front view and lower view.

and the classic definition of watershed includes no topology constraints. Therefore, watershed-based interface may not give good results from a topological point of view. Noise 
in the images can increase topological problems too. An alternative topology-controlled approach might consist of two simultaneous $\lambda$-reconstructions running towards each other, started from the CSF and some marker situated in the white matter. The preference could be done to points where the intensity gradient is lower. It is likely that the two propagation fronts meet in the gradient crest, but we have not tested this method yet. The authors of Refs. [48,49] propose an approach that allows a more precise description of the cortical surface, using cellular complexes. This description, however, which has been developed for a different purpose, makes the treatment of the cortical surface much more difficult, and is not very suited for creating a volumic mesh. These constraints being taken into account, our approach has proven to be very robust. It delivers a volumic representation of the cortical surface with the topology of a hollow sphere. Therefore, it is very suitable for creating a mesh representation of the cortical surface.

\subsection{Separation of hemispheres}

From an anatomical point of view, the two hemispheres are separated by the fissura longitudinalis cerebri, a very deep and narrow furrow. Due to the resolution of the MRI technique, the convolutions that lie at opposite sides of the fissura longitudinalis cerebri seem sometimes to grow together. In fact, they are not really connected. The space between them is too small to appear in the MRI image. Another difficulty is that the hemispheres are physically connected by the corpus callosum, and the division within the corpus callosum can only be done somewhat arbitrarily. By using the component tree to the $X_{\text {CEREBRUM }}$ (see Section 2) searching for two markers, we obtain markers for the two hemispheres, $M=\left\{M_{1}, M_{2}\right\}$. During the second step (the reconstruction of the hemispheres from the markers), it may happen that voxels of one hemisphere are wrongly assigned to the other. This happens due to connections of hemispheres in the MRI images (partial volume effect and corpus callosum), and a dissymetry between both hemispheres, that is occasionally found. To deal with this problem we modify the markers. The markers are cut in both the antero-posterior and up-down directions, so that they have the same projections in the left-right direction. This method follows the form of the fissura longitudinalis and gives satisfactory results even for brains where one of the hemispheres is larger than the other. The hemispheres are obtained by reconstruction from the two markers: $\left\{X_{H L}, X_{H R}\right\}=\lambda$-thickening $(M)$, where $\lambda$ is $g<\infty$, where $g(x)=\operatorname{dist}^{X_{C E R E B R U M}}(x, M)$. Fig. 12 presents an example of the result. Fig. 14 illustrates a case of a marker that has grown into the other hemisphere. The reason for this phenomenon can be found in Fig. 13. The left marker is still present in the lower region of the left hemisphere whereas, in the corresponding region in the right hemisphere, its marker is already completely eroded away. Fig. 15 illustrates the modified markers according to the proposed method. The result of the conditional dilation
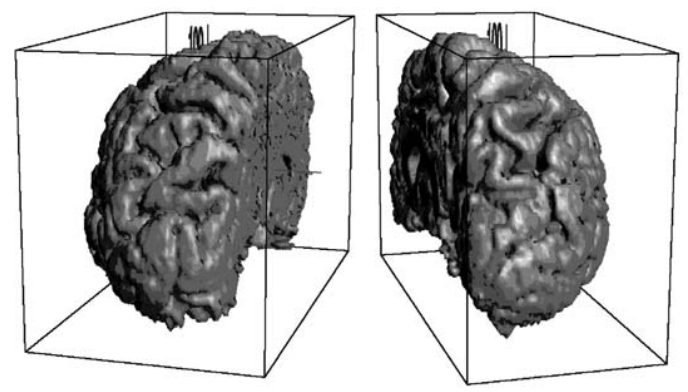

Fig. 12. The separated hemispheres in 3D.

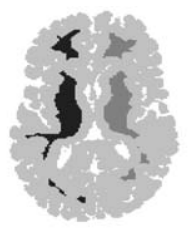

axial view

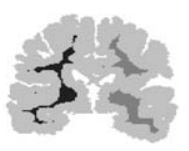

coronal view

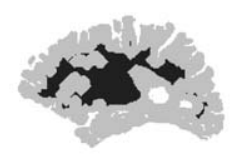

sagittal view
Fig. 13. Example of unmodified marker volumes.

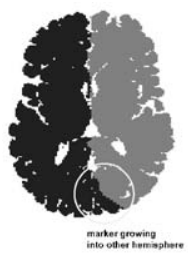

axial view

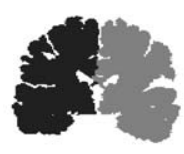

coronal view

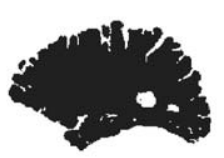

sagittal view
Fig. 14. Example of a marker that grew into the other hemisphere.

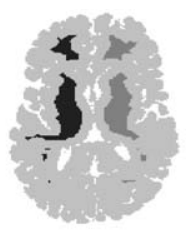

axial view

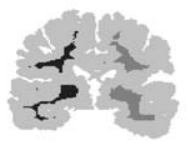

coronal view

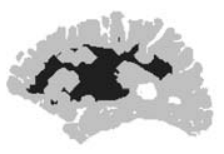

sagittal view
Fig. 15. Modified marker volumes.

of these markers is presented in Fig. 16 and illustrates the improvement provided by the modification of markers. The proposed procedure, applied to several MRI images has proven to be very robust. However, for some MRI images the assignment of a small number of voxels may be 


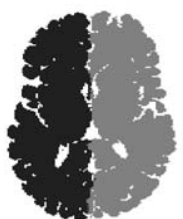

axial view
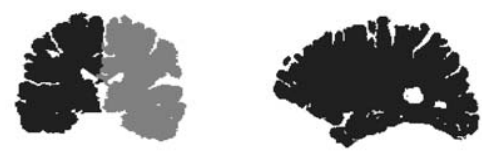

coronal view sagittal view
Fig. 16. Separated hemispheres, obtained from modified markers.

arguable. Especially the precise anatomical division of the two hemispheres is rather subjective. A possible accuracy improvement might be obtained by using methods based on dynamical surfaces (snakes). Nevertheless, considering the extremely complex geometrical form of the cortex, these methods get easily trapped in local minima. Therefore, the segmentation results obtained by the topology-controlled approach proposed here can be used as the initialization of the dynamic surface. The evolution of the surface must be limited to only some little extent to avoid the risk of getting stuck in some local minimum.

\subsection{Skin}

The first step to extract the skin consists in extracting the mask of the head $X_{H E A D}$. This object is simply connected with no holes nor cavities and is obtained as follows. After an initial thresholding and selection of the greatest connected component $X_{10}$ of $\left\{x \mid f(x)>s_{3}\right\}$, where $s_{3}=\mu_{C S F}-\sigma_{C S F}$, we perform a smoothing by a morphological closing: $X_{16}=$ $\varphi_{\Gamma}\left(X_{10}\right), \Gamma$ is a ball of $R=5 \mathrm{~mm}$. Filling of cavities leads to $X_{17}=\operatorname{Fill}\left(X_{16}\right)$. The object $X_{17}$ is too smooth and does not follow correctly the contours of the head. Therefore we propose to add a peeling step eliminating the dark regions (air) introduced by the closing. The closing, however, could not be omitted since its purpose is to close the orifices (ears...) which would have otherwise connected the exterior of the head with the bone, since the air and the bone have similar grey levels. The peeling is only limited to dark points (the air) and up to some maximum distance from the surface of $X_{H E A D}$. A geodesic transform can be used $g(x)=\operatorname{dist}^{\overline{X_{15}}}\left(\overline{X_{17}}\right)$, and the peeling is achieved by $\lambda$-thinning $\left(X_{17}\right)=X_{H E A D}$ where $\lambda: g(x)<R$, where $R$ is the radius used in the closing $(5 \mathrm{~mm}) . X_{H E A D}$ has therefore the correct topology and its surface follows the contour of the head. The skin $X_{\text {SKIN }}$ is obtained in the next step by a thickening of the border of the mask of the head bd $\left(X_{H E A D}\right)$. The thickening is bounded by a maximum distance from the contour and is limited to bright points only, likely to belong to the skin. This restriction is achieved by a geodesic transform $g(x)=\operatorname{dist}^{\overline{X_{15}}}\left(\overline{X_{H E A D}}\right)$. Homotopic thickening of the contour of the head is performed as follows: $X_{S K I N}=\lambda$-thickening $\left(\operatorname{bd}\left(X_{H E A D}\right)\right)$, where $\lambda: g(x)<C$, where $C=6 \mathrm{~mm}$ (an arbitrarily chosen maximum allowed thickness of the skin and epidermis). The
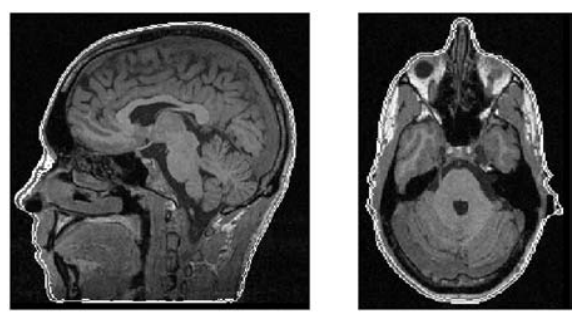

Fig. 17. Contours of the skin $X_{S K I N}$ (in white), sagittal and axial views.

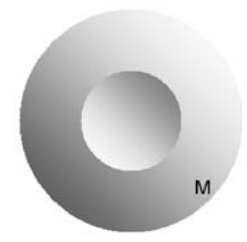

(a)

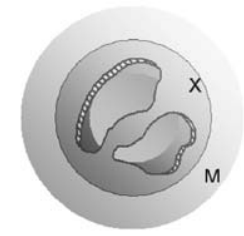

(b)

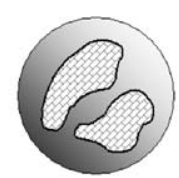

(c)
Fig. 18. Filling of holes on a hollow sphere. (a) the object $M$ is a sphere with one cavity, (b) the object with holes $X$ inside $M$, (c) the holes of $X$ are filled whereas its concavity becomes a cavity.
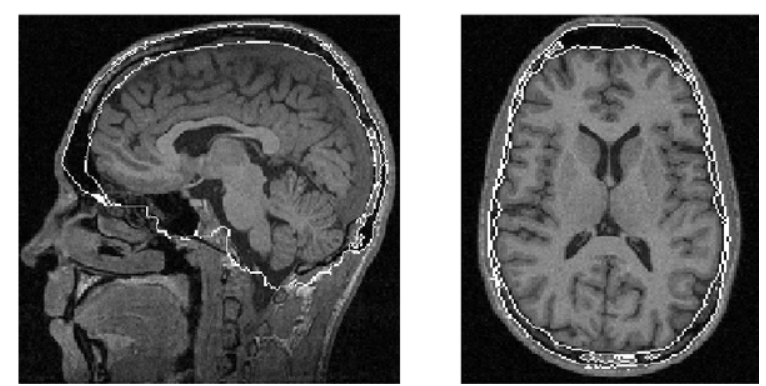

Fig. 19. Contours of the cranium with closed holes (reduced to the skullcap and base), sagittal and axial views.

thickening extends homotopically the head contour inwards. $X_{S K I N}$ has the topology of a hollow sphere and entirely covers the head. The orifices are covered by an one-point-thick surface (Fig. 17).

\subsection{Skull}

The extraction of the skull is subject to two constraints: (i) The resulting object $X_{S K U L L}$ is homotopic to a hollow sphere. Note that this constraint is imposed by the foreseen applications and does not correspond to the reality. It is completely different from the one of Ref. [25] where no precise topology is required. (ii) Focus is given only to the skullcap and the cranium basis whereas the regions situated far from the cortical surface (jaw and sinus) are eliminated. Recall that the result of the segmentation is an individual head model. 

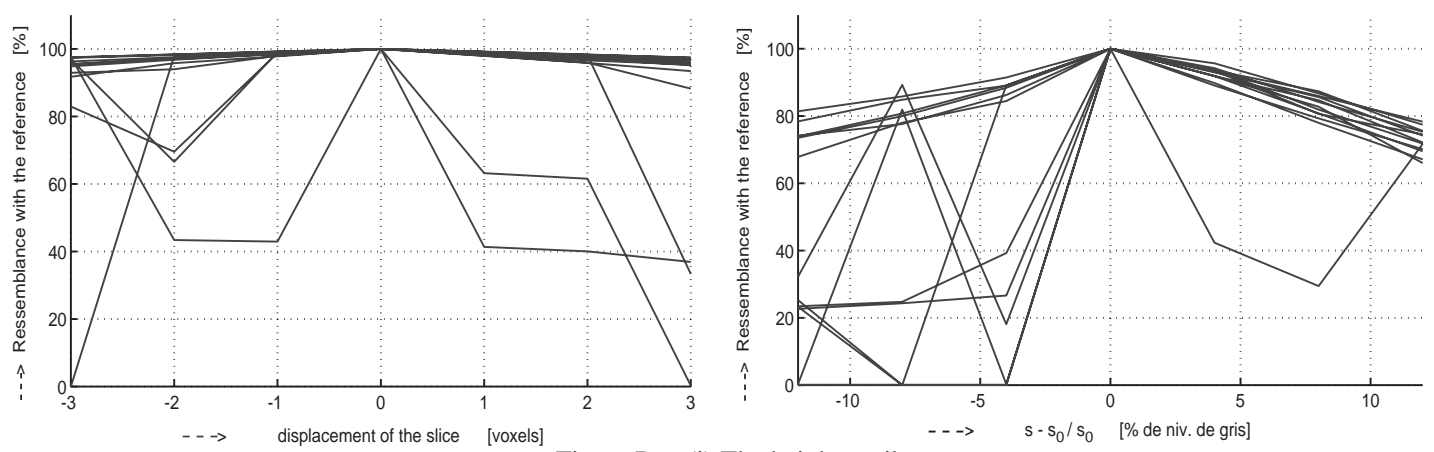

Figure Part (i) The height attribute
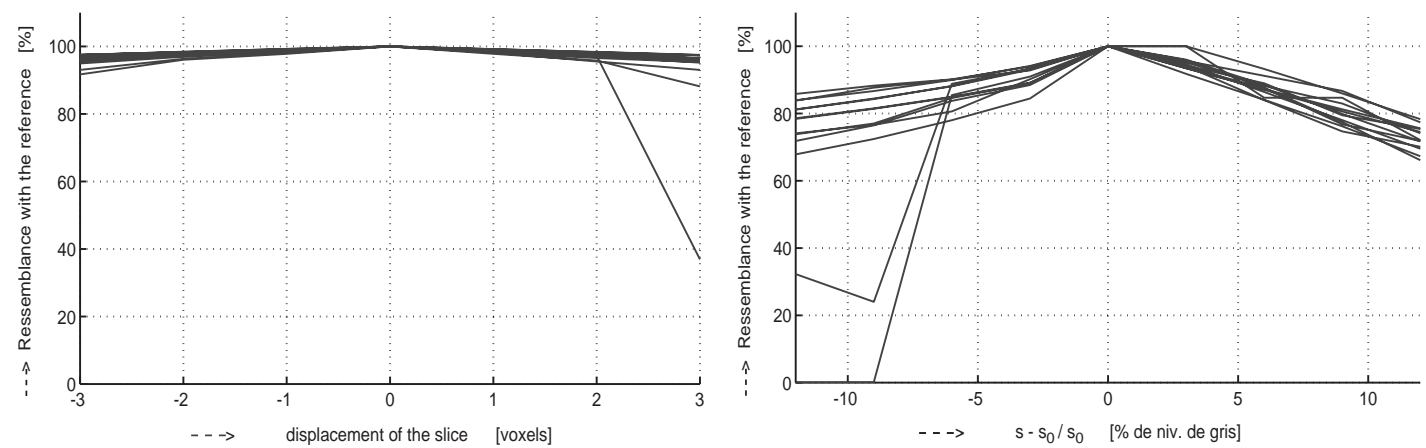

Figure Part (ii) The area attribute
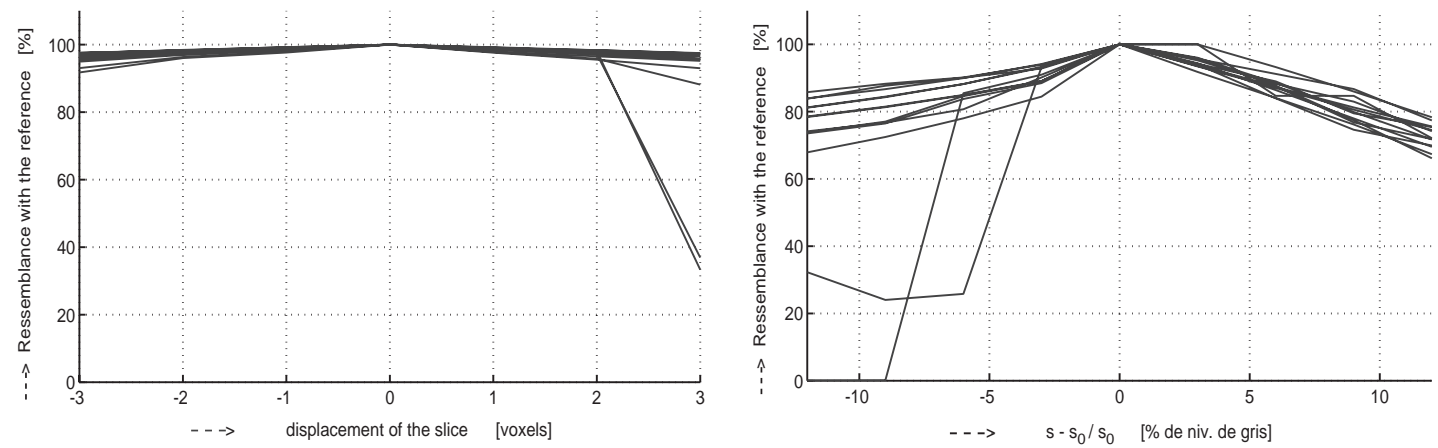

Figure Part (iii) The volume attribute

Fig. 20. Quality of extraction of the cerebellum and the brain stem with respect to the inaccuracy of parameters: manually-found upper extremity of the tegmentum (left column) and thresholding parameter $s$ (right column) when using the height, area or volume attribute (figure Parts i, ii and iii, respectively). Part (i). The height attribute. Part (ii) The area attribute. Part (iii). The volume attribute.

This model is later used as a simulation support for electromagnetic field penetration through various tissues of the head. The sinus and jaw are situated relatively far from the cortex and possess a complex topology. The numerical solution of waveform propagation equations applied to these objects would be useless and overwhelmingly complex. The segmentation proceeds in two steps according to these requirements: a presegmentation step followed by a hole (tunnel) closing.

(1) Presegmentation: masking of the encephalon and the air. We first define $X_{12}=X_{\text {HEAD }} \backslash X_{\text {ENCEPH }}$. Then by thresholding we get $X_{13}=\left\{x \mid x \in X_{12}\right.$ and $\left.f(x)<s_{3}\right\}$, where $s_{3}=\mu_{\text {CORTEX }}-\sigma_{\text {CORTEX }}$. The mask "close to the cortex" is defined as $X_{M A S K}=\delta_{\Gamma}\left(X_{C E R E B R U M} \cup X_{C B}\right)$, where $\Gamma$ is a ball of $R=25 \mathrm{~mm} ; X_{S K U L L}^{\prime}=X_{13} \cap X_{M A S K}$. The mask includes the skullcap, the front and the cranium base. The sinus and the jaw are suppressed.

(2) Hole closing: We use a modified version of the hole closing algorithm of [44]. The new version is based on homotopic deformations controlled by a distance function from the object (see Fig. 18). Let $X$ denote the object submitted to the modified filling algorithm. In the real case $X$ represents the skull. This object has holes-either natural orifices or induced by noise. Filling of these holes will give birth to 
the central cavity occupied by the encephalon. The starting object $M, M \supset X$, has the correct topology (equivalent to a hollow sphere). $M$ is then homotopically deformed towards the desired result. The deformation prevents the creation of holes and preserves the only cavity (Fig. 19).

The hole closing applied on the skull starts with the object $X_{14}=X_{M A S K} \backslash\left(X_{C E R E B R U M} \cup X_{C B}\right)$ containing the skull (with holes). The distance function $d(x)=\operatorname{dist}\left(x, X_{S K U L L}^{\prime}\right)$ controls the hole closing. The resulting object $X_{S K U L L}$ is obtained by homotopic thinning: $X_{S K U L L}=\lambda$-thinning $\left(X_{14}\right)$, where $\lambda$ is $d(x)>0$.

\section{Experiment results}

The segmentation proceeds sequentially. Later stages depend on previous intermediate results. Good robustness is therefore important at every stage since an early error would propagate throughout the entire segmentation process. In this section we evaluate the sensitivity to the parameter values.

\subsection{Parameter estimation and robustness}

The segmentation algorithm uses several parameters. In this section we show experimentally the sensitivity to incorrect parameter values. The sizes of the structuring elements are derived from the anatomical knowledge and the same values could be successfully used on all examples. Markers are selected automatically, and geodesic transformations and reconstruction contribute to the robustness of the method. Grey level parameters are estimated automatically with the $k$-means algorithm, with $k=5$ for five classes (ordered by increasing grey level: air and bone, cerebrospinal fluid, grey matter, white matter, fat). The mean values $\mu_{i}$ and standard deviations $\sigma_{i}$ are calculated for each of the five classes found by the $k$-means. Sometimes (about 30 percent of all cases) the $k$-means fails to identify the cerebrospinal fluid (due to the partial volume effect and the negligible volume occupied by this structure compared to other objects). A manual intervention is therefore needed to verify the result of the $k$-means. Except the positioning of the horizontal slice used to segment the cerebellum and the brain stem, no other user-interaction is needed. These limited interactions are fully reasonable for the foreseen applications. The quality of the result is assessed by introducing a measure $d$ of similarity between two segmented images $f$ and $g$. One of them represents a reference result and the other the tested one. The reference result is obtained with the correct value of the parameter. The values of the other parameters remain fixed and equal to the automatically found ones. Then, the value of tested parameter is subject to alteration with an added error. We assume the following: $g, f: \mathbb{Z}^{3} \rightarrow\{0,1, \ldots, n\}$, where $n$ is the number of objects (same for $g$ and $f$ ) and the set $\{x \mid g(x)=0\}$ (or $\{x \mid f(x)=0\}$ ) is the background for $g$ (respectively for $f$ ). In our case, the segmentation technique guarantees a correct topology and that the number $n$
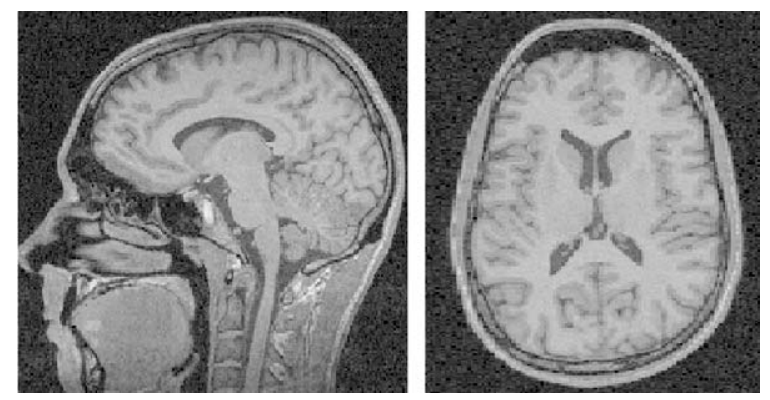

Fig. 21. Example of an input image, sagittal and axial views.
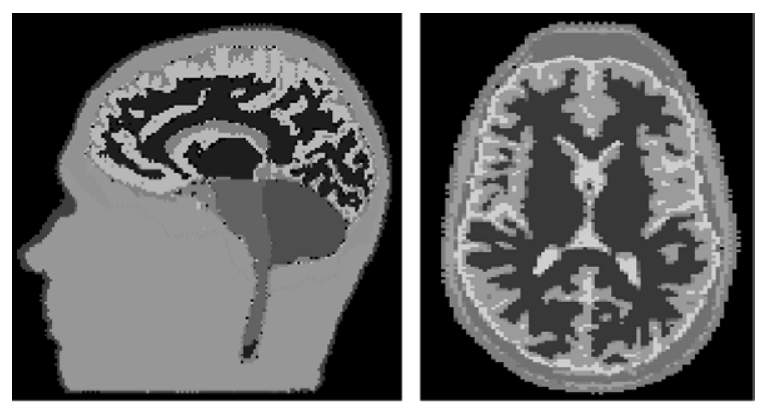

Fig. 22. Result of the segmentation of the image given in Fig. 21.

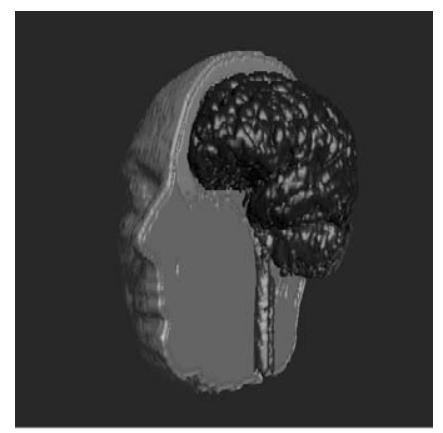

Fig. 23. 3D view of the segmented result.

of resulting objects in $g$ and $f$ is constant. The topology and the number of objects is constant whatever the value of parameter. Therefore, no considerations have to be made about the number of objects or the topology of the result. We propose a similarity measure defined as

$d(f, g)=\frac{N_{A}(f, g)}{N_{D}(f, g)+N_{A}(f, g)}$,

where $N_{A}$ is the number of points associated to the same structure in $f$ and $g$ given by $N_{A}(f, g)=\#\{x \mid f(x)=$ $g(x), f(x) \neq 0, g(x) \neq 0\}$ and $N_{D}$ is the number of points attributed to different structures $N_{D}(f, g)=\#\{x \mid f(x) \neq$ $g(x)\}$. The symbol \# denotes the cardinal of a set. This measure ensures that $d(f, g)=d(g, f), d(f, g) \in[0,1]$, and 


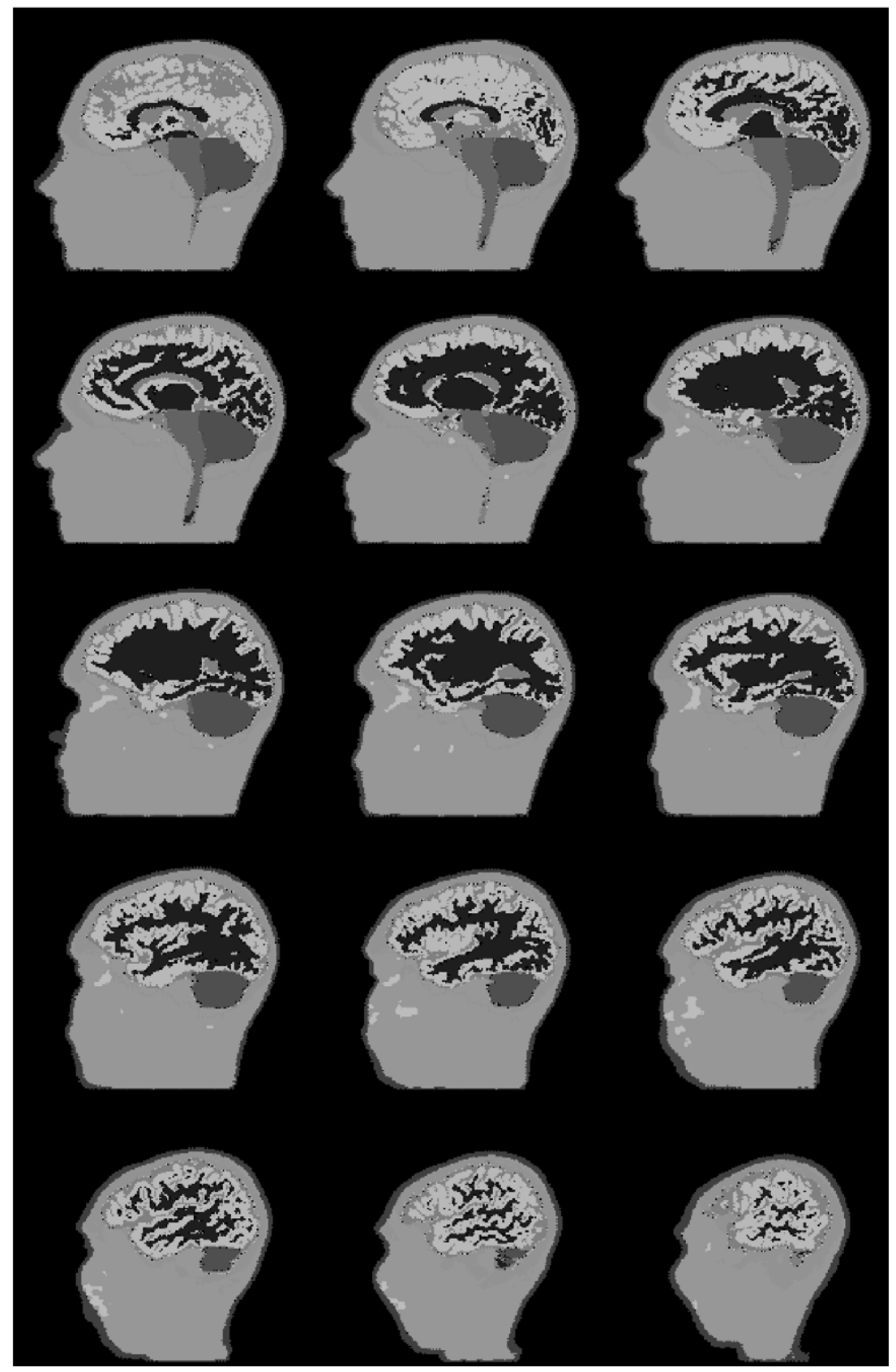

Fig. 24. Set of sagittal slices of the resulting contours (from the center to one side).

yields values close to zero (or one) for low (or high) rate of similarity between $f$ and $g$, respectively.

\subsection{Results}

Two parameters are essential to a successful segmentation, both concerning the identification of the brain stem and cerebellum: the position of the manually given horizon- tal slice delimiting the upper extremity of the tegmentum and the grey-level parameter $s_{1}$ automatically found by the $k$-means analysis. These parameters are critical, since an incorrect value may result in misidentification of the brain stem and cerebellum. The other parameters are not critical; an inaccuracy of the value can only slightly deteriorate the segmentation quality. It may only result in displacement of the contours of the object, and no object misidentification 
may occur. The misidentification is reflected in the similarity measure as a rapid decrease of the correspondence rate, since the number of incorrectly attributed points considerably grows. For a slight displacement of the contours, the values of the similarity measure $d$ remain close to $100 \%$ of correctly attributed points. We show the sensitivity of the result to the error of these two critical parameters. Fig. 20 illustrates the robustness of the brain stem and cerebellum extraction when using the height (Part i), area (Part ii) and volume attribute (Part iii) as criterion for the marker selection (see Section 2). We have traced the segmentation quality by using the measure $d$ for each of the testing MRIs versus a set of erroneous values of the two parameters. Each graphic contains therefore one curve per image. The horizontal axis represents the error introduced to the parameter. The error is calculated as $\left(s-s_{0}\right) / s_{0} .100 \%$, where $s_{0}$ is the correct value. The central value is error-free; the error is positive to the right and negative to the left. The vertical axis shows the similarity rate with respect to the central error-free result (therefore, always $100 \%$ ). As the error grows, the similarity rate decreases. The left-column graphics give the influence of an erroneous displacement of the manually found upper extremity of the tegmentum. The height attribute is not sufficiently robust to an incorrect value. The area-based (respectively the volume-based) results exhibit a good error insensitivity with only one (or two) misidentifications out of 13 tests for the displacement of +3 slices (vertical resolution is $1.5 \mathrm{~mm}$ per slice). The right-column graphics give the sensitivity to the inaccuracy of the thresholding parameter $s_{1}$. Again, the area and volume-based criteria exhibit considerably better robustness than the height-based one. The error equal to $-9 \%$ introduces two misidentification for the area-based extraction. The error of $-6 \%$ and $-8 \%$ introduces one and two misidentifications for the volume-based extraction. A discussion with neurologists revealed that two manual interventions needed to control the segmentation process are acceptable: (1) verification of the clusters found by $k$-means and (2) location of the top extremity of the tegmentum. Indeed, they belong to the interactions that the users are ready to do to speed up the process and get good results. Fig. 21 gives an example of the input data. Fig. 22 gives the segmentation results corresponding to these slices. Fig. 23 gives a 3D rendering whereas Fig. 24 gives a series of slices throughout the entire volume.

\section{Conclusion}

In this paper, we have proposed an original method for the segmentation of 3D MRI scans of the head. Several structures are extracted one by one by using operators from the frameworks of mathematical morphology and digital topology. The advantage of these operators is that they allow to account easily for available knowledge concerning geometry (shape, size, thickness...) of the objects of interest as well as topology, which is a major constraint in the fore- seen application. Despite the anatomical variations between different individuals and the diversity of the input images (acquired by several scanners in various laboratories), we have observed a satisfactory insensitivity to the parameters error limiting thus the necessary manual intervention. The segmentation result is always a partition of the space, i.e. no points remain unlabeled or associated to two structures. A correct 3D topology of the result is always guaranteed by the used approach. Although different works in the literature address already the brain segmentation, the foreseen applications called for other methods focusing to the topology of the result. The originality of the proposed approach is to provide an automatic and robust method devoted to specific applications. The constraints are derived from the application and are guaranteed even if they are not completely true in reality or in the data. The reduced number of parameters, the automatic selection of markers and the use of morphological reconstruction contribute to the increase in robustness. Moreover, this work shows a real application relying on sound theoretical bases in digital topology. The overall execution time necessary for a Sun Ultra 5 to segment a $256 \times 256 \times 160$-point image (i.e. equal to $10 \mathrm{MB}$ of data if coded in eight bits) is $25-35 \mathrm{~min}$. The results have been positively evaluated by medical doctors and electrophysiologists and serve now as individual models for solving equations simulating the electromagnetic wave propagation in head tissues.

\section{References}

[1] J.C. Bezdek, L.O. Hall, L.P. Clarke, Review of MR image segmentation techniques using pattern recognition, Med. Phys. 20 (4) (1993) 1033-1048.

[2] A.P. Zijdenbos, B.M. Dawant, Brain segmentation and white matter lesion detection in MR images, Crit. Rev. Biomed. Eng. 22 (5-6) (1994) 401-465.

[3] L.P. Clarke, R.P. Velthuizen, M.A. Camacho, J.J. Heine, M. Vaidyanathan, L.O. Hall, R.W. Thatcher, M.L. Silbiger, MRI segmentation: methods and applications, J. Magn. Reson. Imaging 13 (3) (1995) 343-368.

[4] E.A. Ashton, K.J. Parker, M.J. Berg, C. Chen, A novel volumetric feature extraction technique with applications to MR images, IEEE Trans. Med. Imaging 16 (4) (1997) 365-371.

[5] M.S. Atkins, B.T. Mackiewich, Fully automatic segmentation of the brain in MRI, IEEE Trans. Med. Imaging 17 (1) (1998) 98-107.

[6] N. Duta, M. Sonka, Segmentation and interpretation of MR brain images: an improved active shape model, IEEE Trans. Med. Imaging 17 (6) (1998) 1049-1062.

[7] S.M. Pizer, D.S. Fritsch, P.A. Ushkevich, V.E. Johnson, E.L. Chaney, Segmentation, registration, and measurement of shape variation via image object shape, IEEE Trans. Med. Imaging 18 (10) (1999) 851-865.

[8] C.A. Davatzikos, J.L. Prince, An active contour model for mapping the cortex, IEEE Trans. Med. Imaging 14 (1) (1995) $65-80$. 
[9] D.L. Collins, P. Neelin, A.C. Evans, Automatic 3D intersubject registration of MR volumetric data in standardized talairach space, J. Comput. Assisted Tomography 18 (2) (1994) 192-205.

[10] F.L. Bookstein, Shape and the information in medical images: a decade of the morphometric synthesis, in: Mathematical Methods in Biomedical Image Analysis, San Francisco, CA, USA, 1996, pp. 2-12.

[11] A.C. Evans, D.L. Collins, C.J. Holmes, in: A.W. Toga, J.C. Mazziotta (Eds.), Computational Approaches to Quantifying Human Neuroanatomical Variability, Academic Press, New York, 1996, pp. 343-361.

[12] Y. Amit, Graphical shape template for automatic anatomy detection with applications to MRI brain scans, IEEE Trans. Med. Imaging 16 (1) (1997) 28-40.

[13] R. Szeliski, S. Lavallée, Matching 3-D anatomical surfaces with non-rigid deformations using octree-splines, Int. J. Comput. Vision 18 (2) (1996) 171-186.

[14] G.E. Christensen, R.D. Rabbitt, M.I. Miller, 3D brain mapping using a deformable neuroanatomy, Phys. Med. Biol. 39 (1994) 609-618.

[15] P.M. Thompson, A.W. Toga, A surface-based technique for warping three-dimensional images of the brain, IEEE Trans. Med. Imaging 15 (4) (1996) 402-417.

[16] J.C. Gee, L. Le Briquer, C. Barillot, D.R. Haynor, Probabilistic matching of brain images, in: Y. Bizais, C. Barillot, R. Di Paola (Eds.), Computational Imaging and Vision: Information Processing in Medical Imaging, Vol. 2432, Kluwer Academic, Dordrecht, 1995, pp. 113-126.

[17] B.M. Dawant, S.L. Hartmann, J.-P. Thirion, F. Maes, D. Vandermeulen, P. Demaerel, Automatic 3D segmentation of internal structures of the head in MR images using a combination of similarity and free-form transformations: part I, methodology and validation on normal subjects, IEEE Trans. Med. Imaging 18 (10) (1999) 909-916.

[18] M.C. Clark, L.O. Hall, D.B. Goldgof, L.P. Clarke, R.P. Velthuizen, M.S. Silbiger, MRI segmentation using fuzzy clustering techniques, IEEE Eng. Med. Biol. 13 (5) (1994) 730-742.

[19] D. Pham, J.L. Prince, C. Xu, A.P. Dagher, An automated technique for statistical characterization of brain tissues in magnetic resonance imaging, Int. J. Pattern Recognition Art. Intell. 11 (8) (1997) 1189-1211.

[20] K. Cheng, J. Lin, C. Mao, The application of competitive hopfield neural network to medical image segmentation, IEEE Trans. Med. Imaging 15 (1996) 560-567.

[21] W.E. Reddick, J.O. Glass, E.N. Cook, T.D. Elkin, J. Deaton, Automated segmentation and classification of multispectral magnetic resonance images of brain using artificial neural networks, IEEE Trans. Med. Imaging 16 (6) (1987) 911-918.

[22] S. Sandor, R. Leahy, Surface-based labeling of cortical anatomy using a deformable model, IEEE Trans. Med. Imaging 16 (1) (1997) 41-54.

[23] J.F. Mangin, V. Frouin, I. Bloch, J. Regis, J. Lopez-Krahe, From 3D magnetic resonance images to structural representations of the cortex topography using topology preserving deformations, J. Math. Imaging Vision 5 (1995) 297-318.

[24] C. Tsai, B. Manjunath, R. Jagadeesan, Automated segmentation of brain MR images, Pattern Recognition 28 (1995) $1825-1837$.
[25] H. Rifai, I. Bloch, S. Hutchinson, J. Wiart, L. Garnero, Segmentation of the skull in MRI volumes using deformable model and taking the partial volume effect into account, Med. Image Anal. 4 (3) (2000) 219-233.

[26] P. Marais, R. Guillemaud, M. Sakuma, A. Zisserman, M. Brady, Visualising cerebral asymmetry, Lect. Notes Comput. Sci.: Visualization Biomed. Comput. 1131 (1996) 411-416.

[27] Y. Liu, R.T. Collins, W.E. Rothfus, Automatic bilateral symmetry (midsagittal) plane extraction from pathological 3D neuroradiological images, Proc. SPIE: Med. Imaging 1998: Image Processing 3338 (1998) 1528-1539.

[28] S. Prima, J.-P. Thirion, G. Subsol, N. Roberts, Automatic analysis of normal brain dissymmetry of males and females in MR images, Lecture Notes Computer Sciences: Proceedings of the International Conference on Medical Image Computing and Computer-Assisted Intervention, Vol. 1496, Springer, Berlin, 1998, pp. 770-779.

[29] S. Prima, S. Ourselin, N. Ayache, Computation of the mid-sagittal plane in 3D brain images, IEEE Trans. Med. Imaging 21 (2) (2002) 122-138.

[30] F. Maes, K. Van Leemput, L.E. DeLisi, D. Vandermeulen, P. Suetens, Quantification of cerebral grey and white matter asymmetry from MRI, Lecture Notes in Computer Sciences: Proceedings of the Second International Conference on Medical Image Computing and Computer-Assisted Intervention, Vol. 1679, Springer, Berlin, 1999, pp. 348-357.

[31] J. Serra, Image Analysis and Mathematical Morphology, Academic Press, London, 1982.

[32] G. Borgefors, Distance transforms in the square grid, in: H. Maître (Ed.), Progress in Picture Processing, Les Houches, Session, Vol. LVIII, 1992, North-Holland, Amsterdam, 1996, (Chapter 1.4) pp. 46-80.

[33] M. Schmitt, J. Mattioli, Morphologie Mathématique, Masson, Paris, 1994.

[34] S. Beucher, Watershed, hierarchical segmentation and waterfall algorithm, in: J. Serra, P. Soille (Eds.), Mathematical Morphology and Its Application to Image Processing, Vol. 2, Kluwer Academic Publishers, Dordrecht, 1994.

[35] P. Hanusse, P. Guillataud, Sémantique des images par analyse dendronique, in: Lyon (Ed.), Eighth Conference on Reconnaissance des Formes et Intelligence Artificielle, AFCET, 1992, pp. 577-588.

[36] C. Vachier, Extraction de caractéristiques, segmentation d'images et morphologie mathématique, Ph.D. Thesis, Ecole des Mines, Paris, 1995.

[37] E.J. Breen, R. Jones, Attribute openings, thinnings and granulometries, Comput. Vision Image Understanding 64 (1996) 377-389.

[38] P. Salembier, A. Oliveras, L. Garrido, Antiextensive connected operators for image and sequence processing, IEEE Trans. Image Process. 7 (1998) 555-570.

[39] J. Mattes, J. Demongeot, Efficient algorithms to implement the confinement tree, Ninth Conference on Discrete Geom. for Computer Imaging, Lecture Notes in Computer Science, Springer, Berlin, 1953 (2000) pp. 392-405.

[40] G. Bertrand, G. Malandain, A new characterization of three-dimensional simple points, Pattern Recognition Lett. 15 (1994) 169-175.

[41] A. Rosenfeld, T.Y. Kong, A. Nakamura, Topology-preserving deformations of two-valued digital pictures, Graphical Models Image Process. 60 (1) (1998) 24-34. 
[42] P. Dokládal, C. Lohou, L. Perroton, G. Bertrand, Liver blood vessels extraction by a 3-D topological approach, in: MICCAI'99, Cambridge, 1999.

[43] P. Dokládal, Grey-scale image segmentation: a topological approach, Ph.D. Thesis, Université Marne la Vallée, Jan 2000.

[44] Z. Aktouf, G. Bertrand, L. Perroton, A 3D hole closing algorithm, Pattern Recognition Lett. 23 (5) (2002) 523-531.

[45] Jean-François Mangin, Mise en corrspondance d'images mdicales 3D multi-modalits multi-individus pour la corrlation anatomo-fonctionnelle crbrale, Ph.D. Thesis, Ecole Nationale Supérieure des Télécommunications, 1995.

[46] J. Serra (Ed.), Image Analysis and Mathematical Morphology, Part II: Theoretical Advances, Academic Press, London, 1988.
[47] T. Géraud, Segmentation des structures internes du cerveau en imagerie par résonance magnétique tridimensionnelle, Ph.D. Thesis, Ecole Nationale Suprieure des Tlcommunications, June 1998. (ENST 98 E 012).

[48] Y. Cointepas, I. Bloch, L. Garnero, A cellular model for multi-objects multi-dimensional homotopic deformations, Pattern Recognition 34 (9) (2001) 1785-1798.

[49] Y. Cointepas, I. Bloch, L. Garnero, A precise segmentation of the cerebral cortex from 3D MRI using cellular model and homotopic deformations, in: H. Yan (Ed.), MR Signal and Image Processing, Marcel Dekker, New York, 2001.

About the Author-PETR DOKLÁDAL has graduated from the Technical University in Brno, Czech Republic, in 1994 as a Telecommunication Engineer. He obtained his Ph.D. degree in January 2000 from the University of Marne la Valle, France, in General Computer Sciences, specialization Image Processing. He has spent ten months, participating in the COMOBIO project, with the Image and Signal Processing Department at the Ecole des Télécommunications in Paris. In November 2000 he joined the Centre of Mathematical Morphology at the School of Mines in Paris as a research engineer. His interests include medical imaging, image segmentation, object tracking and pattern recognition.

About the Author-ISABELLE BLOCH is professor at ENST (Signal and Image Processing Department). She graduated from Ecole des Mines de Paris in 1986, received Ph.D. from ENST Paris in 1990, and the "Habilitation Diriger des Recherches" from University Paris 5 in 1995. Her research interests include 3D image and object processing, 3D and fuzzy mathematical morphology, discrete 3D geometry and topology, decision theory, information fusion in image processing, fuzzy set theory, belief function theory, structural pattern recognition, spatial reasoning, medical imaging.

About the Author-MICHEL COUPRIE received the engineer degree from ESIEE (Noisy-le-grand, France) in 1985 and the Ph.D. degree from the Pierre et Marie Curie University (Paris, France) in 1988. Since 1988 he has been working in ESIEE where he is an Associate Professor. His current research interests include image analysis and discrete mathematics.

About the Author-DANIEL RUIJTERS was born on the 1st of February 1974 in Heerlen, the Netherlands. He contributed to the COMOBIO project at ENST Paris as part of his master thesis. Currently he is working at Philips Medical Systems in the Netherlands. He has received his Diplom Ingenieur degree at the University of Technology (RWTH) Aachen (Germany) in 2002. During his studies he has worked at ELSA in Aachen and at the institute of psychology of the RWTH. His primary research interest areas are medical image processing and $3 \mathrm{D}$ computer vision.

About the Author-RAQUEL URTASUN was born on the 30th of January 1976 in Pamplona, Spain. She received her telecommunication engineer's degree from the Public University of Navarra in 2000. She spent six months participating in the COMOBIO project, with the Image and Signal Processing Department at the Ecole des Télécommunications in Paris. In October 2000 she joined the EPFL in Lausanne, Switzerland, as a postgraduate student. She is currently doing her Ph.D. in the CVLab-EPFL. Her interests include computer vision, medical imaging and pattern recognition.

About the Author-LINE GARNERO was born in France, on September 4,1955. She received the Doctorat de 3ème Cycle and the Doctorat d'Etat from the University of Paris XI (Orsay) in 1981 and 1987, respectively. She is Directeur de Recherche at the CNRS (National Center of Scientific Research). She worked at the Institute of Optics in Orsay from 1981 to 1996. Her research field was the image reconstruction techniques for microwave, X-ray or g tomography. She works now in the laboratory of Cognitive Neurosciences and Cerebral Imaging in La Salpêtrière Hospital, Paris, where her main research concerns the reconstruction of brain electrical activity from electroencephalographic (EEG) or magnetoencephalographic (MEG) data, and the fusion IRMf/EEG/MEG. 